\title{
New approach to wireless data communication in a propagation environment
}

\author{
Wojciech P. Hunek ${ }^{1, *}$, and Pawet Majewski ${ }^{2}$ \\ ${ }^{1,2}$ Opole University of Technology, Institute of Control Engineering, Prószkowska 76, 45-758 Opole, Poland
}

\begin{abstract}
This paper presents a new idea of perfect signal reconstruction in multivariable wireless communications systems including a different number of transmitting and receiving antennas. The proposed approach is based on the polynomial matrix $S$-inverse associated with Smith factorization. Crucially, the above mentioned inverse implements the so-called degrees of freedom. It has been confirmed by simulation study that the degrees of freedom allow to minimalize the negative impact of the propagation environment in terms of increasing the robustness of whole signal reconstruction process. Now, the parasitic drawbacks in form of dynamic ISI and ICI effects can be eliminated in framework described by polynomial calculus. Therefore, the new method guarantees not only reducing the financial impact but, more importantly, provides potentially the lower consumption energy systems than other classical ones. In order to show the potential of new approach, the simulation studies were performed by author's simulator based on wellknown OFDM technique.
\end{abstract}

\section{Introduction}

As we all know the environment in which we live and move has a huge impact on our behavior. In fact, without proper air conditions we cannot to function normally. That is why it is so important to be able to determine the positive or negative impact of the environment on our actions. In this paper, a special attention has been paid to the parasitic influence of the environment on the wireless data transmission process. Through an application of different tools we can effectively identify and reduce the noise impact under various engineering tasks including a multivariable real perfect signal reconstruction problem. A recently introduced new method of signal recovery is presented and confirmed by simulation examples here.

Today's wireless data communication standards are already well developed. There are a number of solutions that allow for balanced transmission of information in order to achieve a significant bandwidth throughput (for example WiMAX, WiFi 802.11n, DVB-T or LTE/LTE Advanced standards), the several of them implement the well-known OFDM mechanism [1,2]. The use of multivariability, which is a response to the paradigm of achieving a significant throughput, entails additional problems such as inter-channel (ICI) and inter-symbol interference (ISI). Notwithstanding, the basic method of independent separate between channels is the usage, for instance, the commonly practiced tool in form of SVD factorization [3-7]. However, all of those methods suffer from the parasitic effect of the environment in the form of noise. This paper presents the results of simulation studies using a new signal reconstruction method dedicated to propagation environments described by nonsquare polynomial matrices. A new approach implies the use of appropriate tools associated with systems described by matrix polynomial framework. A key role plays here the Smith-factorized the polynomial matrix $S$ inverse, which implements the so-called degrees of freedom [8-15]. As is shown by simulation studies, the new idea can restrain the negative influence of the propagation environment on the process of signal reconstruction/recovery.

The paper is organized in the following manner. A system representation is given in Section 2. Next unit includes the notion of $S$-inverse. A new approach to problem of signal recovery in MIMO telecommunications systems is presented in Section 4. A simulation study of Section 5 confirms a big potential of new method. Finally, the conclusions are shown at the end of the paper.

\section{System representation}

In this paper the simulation studies of wireless data communications were carried out using a system with $N_{T}$-transmitting antennas and $N_{R}$-receiving antennas described by the polynomial matrix of the form [16]

$$
\underline{\mathbf{C}}_{N_{R} \times N_{T}}\left(q^{-1}\right)=\left[\begin{array}{ccc}
\underline{\mathbf{c}}_{11}\left(q^{-1}\right) & \cdots & \underline{\mathbf{c}}_{1 n}\left(q^{-1}\right) \\
\vdots & \ddots & \vdots \\
\underline{\mathbf{c}}_{m 1}\left(q^{-1}\right) & \cdots & \underline{\mathbf{c}}_{m n}\left(q^{-1}\right)
\end{array}\right]
$$

where operator $q^{-1}$ is the background shift operator. And polynomials describing individual cells of $\underline{\mathbf{C}}\left(q^{-1}\right)$ matrix are finite ipulse response type (FIR). For such described

* Corresponding author: w.hunek@po.opole.pl 
environment model, the signal transition through the propagation path is illustrated in the Fig. 1. Therefore, the signal transition equation can be written according to the formula [16]

$$
\mathbf{R}^{\prime}(t)=\underline{\mathbf{C}}\left(q^{-1}\right) \mathbf{S}(t)+\zeta(t),
$$

where $\mathbf{R}^{\prime}(t), \mathbf{S}(t)$ and $\zeta(t)$ are the vectors of the noisied received signal, transmitted signal and noise signal, respectively. In simulation studies we also assumed that the analyzed disturbance is an uncorrelated white noise with zero-mean value.

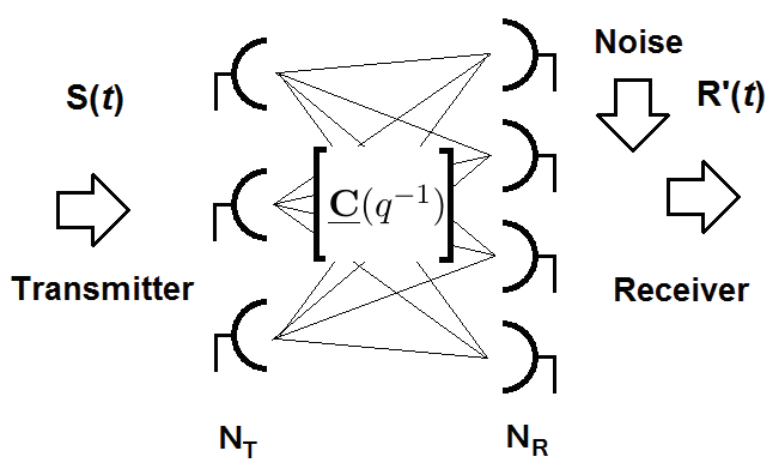

Fig. 1. Model of wireless signal path [16].

Because fo the new method is based on a polynomial approach, the next section presents alternative tools that are equivalent to those commonly used in the classical reconstruction processes, where the propagation environments are described by parametric matrices [1719].

\section{Polynomial matrix S-inverse}

Due to the fact that in the new approach the signal reconstruction process uses the polynomial matrices to described a propagation parameters, the well-known SV decomposition for channel separation cannot be applied. On the other hand, in the literature we can find a several solutions related to SVD factorization in form, e.g., PSVD (Polynomial-SVD) [20-22]. In this paper a new approach to perfect signal recovery is presented, which is based on so-called polynomial matrix $S$-inverse. This tool does not only provide a channel separation using the so-called Smith factorization, but penetrates the whole process of signal reconstruction. The Smith factorization for the matrix $\underline{\mathbf{C}}\left(q^{-1}\right)$ being of the full normal rank with FIR-polynomials is the following

$$
\underline{\mathbf{C}}\left(q^{-1}\right)=\underline{\mathbf{U}}\left(q^{-1}\right) \underline{\sum}\left(q^{-1}\right) \underline{\mathbf{V}}\left(q^{-1}\right),
$$

where the unimodular matrices $\underline{\mathbf{V}}\left(q^{-1}\right)$ and $\underline{\mathbf{U}}\left(q^{-1}\right)$, as equivalent to the parameter signal reconstruction approach, are precoder and equalizer, respectively [20], whilst matrix $\Sigma\left(q^{-1}\right)$ includes eigenvalues of matrix $\underline{\mathbf{C}}\left(q^{-}\right.$ $\left.{ }^{1}\right)$. Taking into account this factorization we obtain right and left polynomial matrix $S$-inverses [8]

$$
\underline{\mathbf{C}}^{\mathrm{R}}\left(q^{-1}\right)=\underline{\mathbf{V}}^{-1}\left(q^{-1}\right) \Sigma^{\mathrm{R}}\left(q^{-1}\right) \underline{\mathbf{U}}^{-1}\left(q^{-1}\right),
$$

$$
\underline{\mathbf{C}}^{\mathrm{L}}\left(q^{-1}\right)=\underline{\mathbf{V}}^{-1}\left(q^{-1}\right) \Sigma^{\mathrm{L}}\left(q^{-1}\right) \underline{\mathbf{U}}^{-1}\left(q^{-1}\right) .
$$

Due to the fact that in the presented in the paper the simulated studies consider the telecommunications systems with a greater number of receiving antennas than transmitting ones $\left(N_{T}<N_{R}\right)$, our attention is focused only on nonunique left inverses. This results that the matrix $\Sigma\left(q^{-1}\right)$, which is depicted in Eqn. (5), is of the following form [23]

$$
\underline{\Sigma}^{\mathrm{L}}\left(q^{-1}\right)=\left[\underline{\mathbf{D}}_{N_{T} \times N_{T}}\left(q^{-1}\right) \quad \underline{\mathbf{M}}_{N_{T} \times\left(N_{R}-N_{T}\right)}\left(q^{-1}\right)\right],
$$

where matrix $\underline{\mathbf{M}}\left(q^{-1}\right)$ contains the crucial degrees of freedom. As has already been confirmed by formal proof [16], in a classical approach, where the Moore-Penrose inverse associated with SVD factorization is commonly used for nonsquare systems, a corresponding to the matrix $\Sigma^{\mathrm{L}}\left(q^{-1}\right)$ structure has zero - degrees of freedom [20]. Therefore in this case we cannot influence the signal perfect recovery through an application of special selected degrees of freedom. The next section presents a new issue concerning the multivariable signal perfect reconstruction including Smith factorization of $\underline{\mathbf{C}}\left(q^{-1}\right)$.

\section{New approach to wireless signal reconstruction in polynomial matrix environment}

Having the background and the necessary tools needed in the new approach to the reconstruction process we can finally go through the description of the main issue of the paper. Let the signal pass through propagation environment described by Eqn. (1) defines the formula (2). After using the Smith factorization, for the purpose of the ICI cancellation, we obtain

$$
\mathbf{R}^{\prime}(t)=\underline{\mathbf{U}}\left(q^{-1}\right) \underline{\sum}\left(q^{-1}\right) \underline{\mathbf{V}}\left(q^{-1}\right) \mathbf{S}(t)+\zeta(t) .
$$

After applying the left polynomial $S$-inverse of Eqn. (5), we receive the reconstructed signal form

$$
\begin{aligned}
\mathbf{S}(t)= & \underline{\mathbf{V}}^{-1}\left(q^{-1}\right) \underline{\Sigma}^{\mathrm{L}}\left(q^{-1}\right) \underline{\mathbf{U}}^{-1}\left(q^{-1}\right) \mathbf{R}^{\prime}(t) \\
& -\underline{\mathrm{V}}^{-1}\left(\mathrm{q}^{-1}\right) \underline{\Sigma}^{\mathrm{L}}\left(\mathrm{q}^{-1}\right) \underline{\mathrm{U}}^{-1}\left(\mathrm{q}^{-1}\right) \zeta(\mathrm{t}),
\end{aligned}
$$

or, after some assumptions [16], as follows

$$
\begin{gathered}
\mathbf{S}(t)+\underline{\mathbf{V}}^{-1}\left(q^{-1}\right) \underline{\Sigma}^{\mathrm{L} 1}\left(q^{-1}\right) \underline{\mathbf{U}}^{-1}\left(q^{-1}\right) \zeta(t) \\
\quad=\underline{\mathbf{V}}^{-1}\left(q^{-1}\right) \underline{\Sigma}^{\mathrm{L}}\left(q^{-1}\right) \underline{\mathbf{U}}^{-1}\left(q^{-1}\right) \mathbf{R}(t) \\
+\underline{\mathbf{V}}^{-1}\left(q^{-1}\right) \underline{\Sigma}^{\mathrm{L} 2}\left(q^{-1}\right) \underline{\mathbf{U}}^{-1}\left(q^{-1}\right) \zeta(t),
\end{gathered}
$$

where $\mathbf{R}(t)$ is the perfect obtained signal, whilst $\sum^{\mathrm{L} 1}\left(q^{-1}\right)$ $\neq \Sigma^{\mathrm{L} 2}\left(q^{-1}\right)$ and $\Sigma^{\mathrm{L}}\left(q^{-1}\right)$ can be any. It is crucial, that trough an application of Eqn. (9) we would like to delete any noises in terms of selection of appropriate degrees of freedom $\underline{\mathbf{M}}\left(q^{-1}\right)$. Therefore, the performance index is determined in the following form $[24,25]$

$$
\underline{\mathbf{M}}_{\mathrm{opt}}\left(q^{-1}\right)=\arg \min _{\underline{\mathbf{M}}\left(q^{-1}\right)} \sum_{t=0}^{N-1}\left\{\left[\mathbf{S}^{\prime}(t)\right.\right.
$$




$$
\left.-\mathbf{S}(t)]^{\mathrm{T}}\left[\mathbf{S}^{\prime}(t)-\mathbf{S}(t)\right]\right\},
$$

which was the basis for genetic algorithm to obtain the optimal degrees of freedom. The goal of the optimization task is receive the smallest difference between the transmitted $\mathbf{S}(t)$ and reconstructed $\mathbf{S}^{\prime}(t)$ signals. The big potential of the new approach shows next section.

\section{Simulation study}

In the simulation studies, a system containing two transmiting antennas and three receiving antennas $\left(N_{T}=\right.$ $2, N_{R}=3$ ) is considered. The parameters describing the propagation properties of the environment are described by the hypothetical polynomial matrix of the form

$$
\underline{\mathbf{C}}\left(q^{-1}\right)=\left[\begin{array}{cc}
0.8+0.4 q^{-1} & 0.8 \\
1-0.6 q^{-1}+0.2 q^{-2} & 1-0.6 q^{-1} \\
1+0.4 q^{-1} & 0.8
\end{array}\right] .
$$

After applying the new method to the process of signal reconstruction, the matrix (11) is factorized and inversed finally to obtain

$$
\begin{aligned}
& \underline{\mathbf{U}}^{-1}\left(q^{-1}\right)=\left[\begin{array}{ccc}
1 & 0 & \cdots \\
1 & 0 & \cdots \\
-1.25-1.75 q^{-1}+2.5 q^{-2} & 1 & \cdots
\end{array}\right. \\
& \left.\begin{array}{lc}
\cdots & 0 \\
\cdots & -1 \\
\cdots & 2.5 q^{-1}-2.5 q^{-2}
\end{array}\right],
\end{aligned}
$$

and

$$
\underline{\mathbf{V}}^{-1}\left(q^{-1}\right)=\left[\begin{array}{cc}
0 & -5 \\
1.25 & 5+2.5 q^{-1}
\end{array}\right],
$$

whilst crucial in the new approach a block matrix containing the degrees of freedom is a form

$$
\underline{\Sigma}\left(q^{-1}\right)=\left[\begin{array}{lll}
1 & 0 & \underline{\mathbf{M}}_{11}\left(q^{-1}\right) \\
0 & 1 & \underline{\mathbf{M}}_{21}\left(q^{-1}\right)
\end{array}\right] .
$$

For the purpose of simulating studies of wireless data transmission an authors' simulator, which implements the new signal reconstruction method, was developed in the Matlab environment. This tool, besides using the new method, takes into account the widely used mechanism OFDM for increasing the transmission of data. However, due to space limitation reasons the simulator is not discussed here in detail (for more information see Ref. [16]). The procedure allows to select the optimal degrees of freedom, which were determined using a genetic algorithm based on the performance index (10). Moreover, said tool also give us the ability to graphical presentation the transmitted bits in the form of constellation maps.

In order to show the obtained results, the basic information related to data transmission, should be given (Table 1).

The simulation studies were performed as follows. For the considered matrix (11) describing the dynamics

of the environment, the transmission of individual bits was simulated.
Table 1. Data transmission parameters.

\begin{tabular}{|c|c|}
\hline Parameter & Value \\
\hline Number of transmitting antennas & 2 \\
\hline Number of receiving antennas & 3 \\
\hline Number of subcarriers & 16 \\
\hline Type of constellation & $64-\mathrm{QAM}$ \\
\hline Number of transmitted bits & 103776 \\
\hline
\end{tabular}

In this scenario the different levels of noise were taking into account, which, according to Eqn. (2), blurred the received signal. Basis on the selected degrees of freedom the Bit Error Rate (BER) index was calculated. The BER index results of new method and classical one (i.e., the approach where the degrees of freedom of the matrix $\Sigma\left(q^{-1}\right)$ of Eqn. (14) are zero) were compared separately. For such studies, the comparative results are shown in Fig. 2. The constellation points obtained from the signal reconstruction process for the classical approach and the new method are presented in the Figs. 3 and 4, respectively.

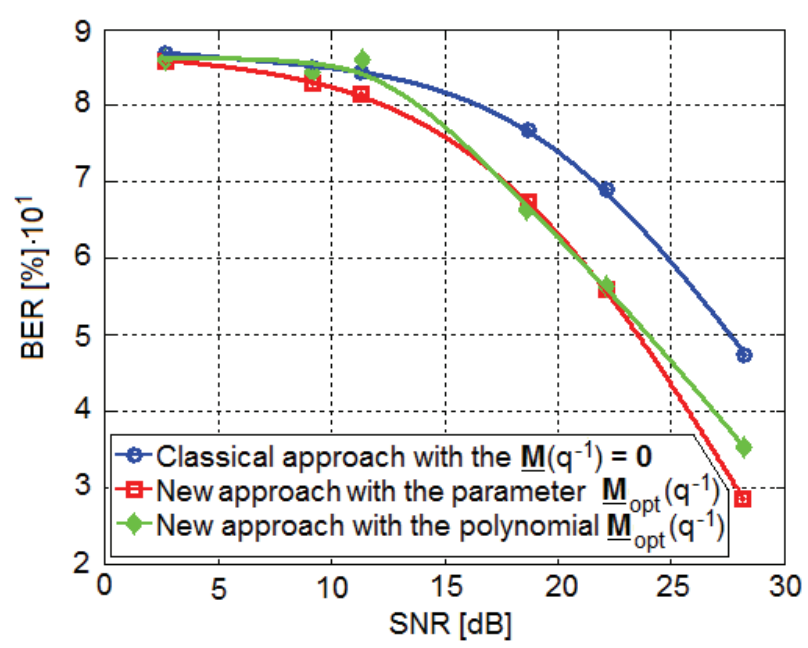

Fig. 2. BER vs. SNR.

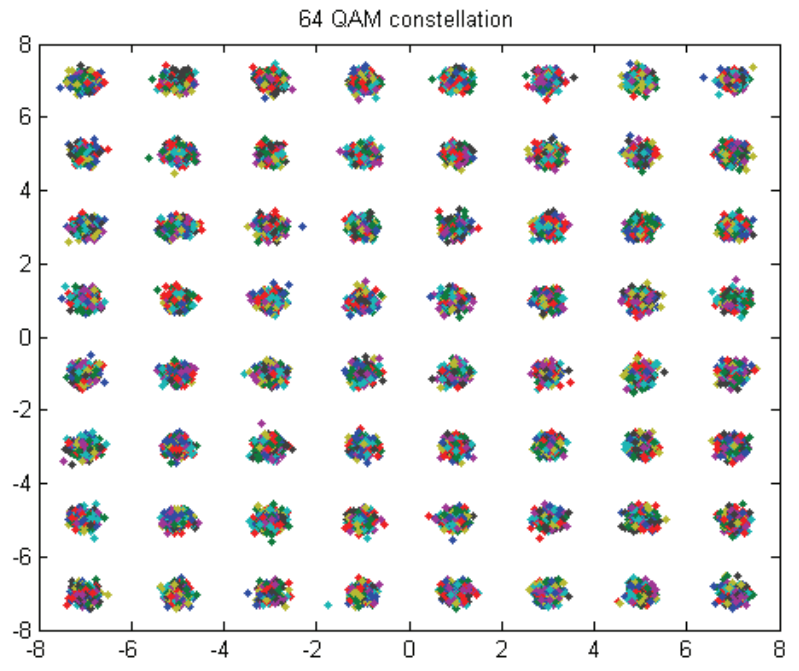

Fig. 3. Classical approach with $\mathrm{SNR}=28 \mathrm{~dB}, \mathrm{BER} 47 \%$. 


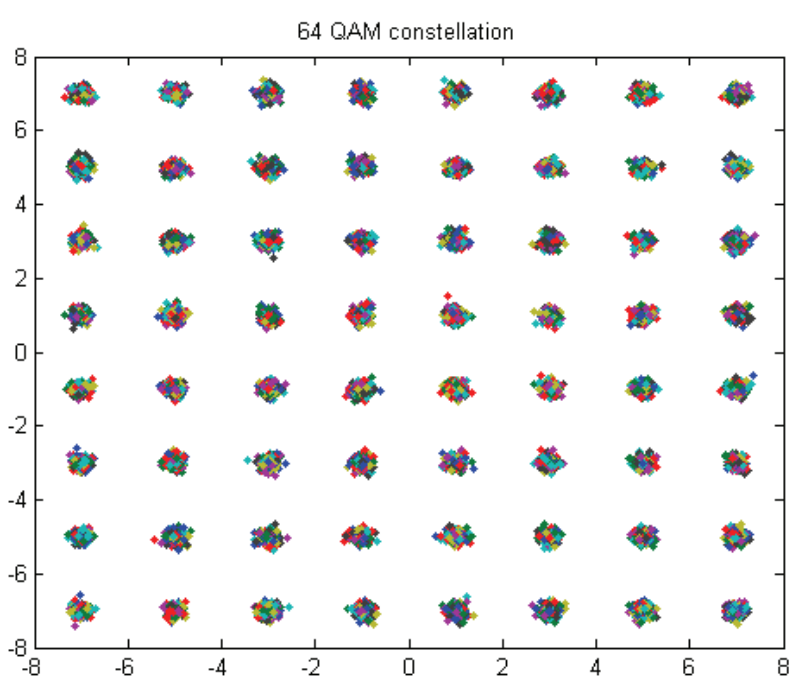

Fig. 4. New approach with $\mathrm{SNR}=28 \mathrm{~dB}$, BER $27 \%$.

\section{Conclusions}

This paper presents a new method for signal reconstruction process. A signal recovery technique based on polynomial matrix description of propagation properties of the natural environment has been proposed. A new approach implements the polynomial matrix $S$ inverse, which contains the crucial degrees of freedom. The superiority of the new method over classical solutions was proven by simulation studies.

\section{References}

1. H. Shu, E. P. Simon, L. Ros, Sig. Proc.117 (2015)

2. D. Mattera, M. Tanda, Dig. Sig. Proc. 49 (2016)

3. O. Nibouche, J. Jiang, Dig. Sig. Proc. 23 (2013)

4. M. Nagahara, IEEE Sig. Proc. Let. 22 (2015)

5. Q. H. Spencer, A. Lee Swindlehurst, M. Haardt, IEEE Tran. on Sig. Proc. 52 (2004)

6. W.P. Hunek, Contribution of a new matrix Hinverse to stabilizing the perfect control for LTI MIMO state-space systems, Proc. of the 21 st Int. Conf. on Met. and Mod. in Auto. and Rob. (MMAR), 64-69 (2016)

7. W.P. Hunek, New SVD-based matrix H-inverse vs. minimum-energy perfect control design for statespace LTI MIMO systems, Proc. of the 20th IEEE Int. Conf. on Sys. The Cont. and Comp. (ICSTCC), 14-19 (2016)

8. W.P. Hunek, Towards a General Theory of Control Zeros for LTI MIMO Systems (Opole Univ. of Tech. Press 2011)

9. W.P. Hunek, K.J. Latawiec, Int. J. Appl. Math. Comp. Sci. 21 (2011)

10. W.P. Hunek, K.J. Latawiec, Cont. Cyb. 38 (2009)

11. W.P. Hunek, Bul. Pol. Aca. Sci. - Tech. Sci. 59 (2011)

12. W.P. Hunek, K.J. Latawiec, P. Majewski, P. Dzierwa, An application of a new matrix inverse in stabilizing state-space perfect control of nonsquare LTI MIMO systems, Proc. of the 19th IEEE Int. Conf. on Met. and Mod. in Auto. and Rob. (MMAR), 451-455 (2014)

13. W.P. Hunek, K.J. Latawiec, R. Stanisławski, M. Łukaniszyn, P. Dzierwa, A new form of a $\sigma$-inverse for nonsquare polynomial matrices, Proc. of the 18th Int. Conf. on Met. and Mod. in Auto. and Rob. (MMAR), 282-286 (2013)

14. W.P. Hunek, An application of polynomial matrix $\sigma$ inverse in minimum-energy state-space perfect control of nonsquare LTI MIMO systems, Proc. of the 20th Int. Conf. on Met. and Mod. in Auto. and Rob. (MMAR), 252-255 (2015)

15. W.P. Hunek, P. Dzierwa, ITC 43 (2014)

16. P. Majewski, Research towards increasing the capacity of wireless data communication using inverses of nonsquare polynomial matrices, Doc. diss. (to appear) (2017)

17. D. Sellathambia, J. Srinivasanb, S. Rajaramc, Hardware implementation of adaptive SVD beamforming algorithm for MIMO-OFDM systems, Proc. of Int. Conf. on Des. and Man, (IConDM'2013), Proc. Eng. 84-93 (2013)

18. M. Sadek, A. Tarighat, A. H. Sayed, IEEE Tran. on Wire. Com., 6 (2007)

19. E.K.S. Au, S. Jin, M.R. McKay, W.H. Mo, IEEE Tran. Wire. Com. 7 (2008)

20. N. Moret, A. Tonello, S. Weiss, MIMO precoding for filter bank modulation systems based on PSVD, Proc. of the 73rd IEEE Veh. Tech. Conf. (VTC'2011), 1-5 (2011)

21. I.A. Akhalaghi, H. Khoshbin, Int. J. Tomo. Stat. 11 (S09) (2009)

22. H. Zamiri-Jafarian, M. Rajabzadeh, A polynomial matrix SVD approach for time domain broadband beamforming in MIMO-OFDM systems, Proc. of the 67th IEEE Veh. Tech. Conf. (VTC'2008), 802-806 (2008)

23. P. Majewski, Sci. Bul. Opole Univ. of Tech. 69 (2013)

24. W.P. Hunek, P. Majewski, Perfect reconstruction of signal - a new polynomial matrix inverse approach, EURASIP J. Wir. Com. and Net., (under review) (2017)

25. W.P. Hunek, P. Majewski, Przegląd Elekrotechniczny 93 (2017) (in Polish) 